\title{
Technical note: Acquisition of CT models for radiotherapy applications with reduced tube heating
}

\author{
James M. Balter ${ }^{a)}$ and Kwok L. Lam \\ Department of Radiation Oncology, University of Michigan Health Systems, Ann Arbor, Michigan 48109
}

(Received 3 November 2000; accepted for publication 17 January 2001)

\begin{abstract}
The potential for changing computed tomography (CT) protocols to provide data sets that generate high quality digitally reconstructed radiographs (DRRs) from scans with very low tube currents is demonstrated. DRRs were generated from CT data acquired with slice thickness of 1,3 , and $5 \mathrm{~mm}$, using high current to reduce noise in axial images. These DRRs were compared to one generated from a CT scan acquired using $1 \mathrm{~mm}$ aperture and very low $(10 \mathrm{~mA})$ current. The DRR generated via this technique is comparable to that generated with high current and $1 \mathrm{~mm}$ aperture, and higher resolution than from the 3 and $5 \mathrm{~mm}$ CT scans. (C) 2001 American Association of Physicists in Medicine. [DOI: 10.1118/1.1354999]
\end{abstract}

Key words: computed tomography, CT simulation, three-dimensional treatment planning, digitally reconstructed radiographs

The use of computed tomography (CT) for establishing patient models in radiotherapy is steadily increasing, driven both by the advent of conformal and intensity-modulated treatment techniques, as well as the increased prevalence of CT simulation. CT-based patient models generally must meet two requirements. They must provide sufficient image quality to permit discrimination of soft tissue anatomy. In addition, they must have sufficient spatial resolution to permit generation of reference digitally reconstructed radiographs (DRRs) that can be used to aid in precision patient setup. ${ }^{1,2}$ These combined demands would suggest the need to provide CT scanners for radiotherapy that are capable of generating thin cuts with high beam current to provide axial images with reduced noise. The result is the need for systems that can handle high rates of tube heating, thus increasing the cost of CT systems for radiotherapy. In addition, the time needed to acquire CT volumes for high resolution definition of primarily bony landmarks presents complications in building accurate models of soft tissue in the thorax and abdomen, where ventilatory immobilization and/or gating is preferential.

Decreasing the tube current during image acquisition decreases the number of photons detected by the detectors, and thus reduces the signal to noise ratio (SNR) of the reconstructed data. Each pixel in a generated DRR, however, is the integral, or weighted sum, through many CT voxels. The weighted summation improves the SNR of the DRR compared to the axial images and can potentially compensate for the decrease in SNR from the reduced tube current. Thus, it should be possible to generate DRRs with acceptable SNR from axial images acquired with reduced tube current over that conventionally accepted.

Based on this premise, it is conceivable that CT scanning protocols can be optimized for radiotherapy to provide the required data for both structure (and density map) definition as well as high resolution DRR generation, but with reduced demands on heat loading. By acquiring axial or helical data at high resolution but with reduced current, it is possible to generate high resolution DRRs with image quality comparable to those generated using high current axial images. These data can exist in conjunction with a lower resolution scan acquired at higher current, to be used in structure definition and density mapping for treatment planning.

An anthropomorphic chest phantom was scanned using a third generation CT scanner (CT/I, GE Medical Systems, Milwaukee, WI). An initial acquisition involved acquiring axial images with $1 \mathrm{~mm}$ aperture and slice separation. Technique was selected to minimize noise in the axial images $(140 \mathrm{kV}, 340 \mathrm{~mA})$. Two volumes of axial images were then acquired using techniques similar to those found in clinical practice for CT simulation. The techniques used for these second and third volumes were $120 \mathrm{kV}$ and $180 \mathrm{~mA}$, with slice thickness of $3 \mathrm{~mm}$ for the second volume and $5 \mathrm{~mm}$ for the third (Table I).

An additional volume was acquired using a new technique. For this scan, axial images were acquired using $1 \mathrm{~mm}$ aperture and slice separation. The maximum $\mathrm{kV}$ available $(140 \mathrm{kV})$ was selected, as well as the minimum $\mathrm{mA}$ setting (10 $\mathrm{mA})$. The goal was to select the technique that provided the highest $\mathrm{x}$-ray quanta production efficiency.

Using these data, a series of DRRs were generated using a conventional CT simulation system (AdvantageSim, GE Medical Systems, Milwaukee, WI) and presented for comparison (Figs. 1-4).

Comparison of techniques 1-3 shows the impact of in-

TABLE I. CT scanning techniques evaluated.

\begin{tabular}{cccc}
\hline \hline Technique & $\mathrm{kV}$ & $\mathrm{mA}$ & Slice thickness $(\mathrm{mm})$ \\
\hline 1 & 140 & 340 & 1 \\
2 & 120 & 180 & 3 \\
3 & 120 & 180 & 5 \\
4 & 140 & 10 & 1 \\
\hline \hline
\end{tabular}




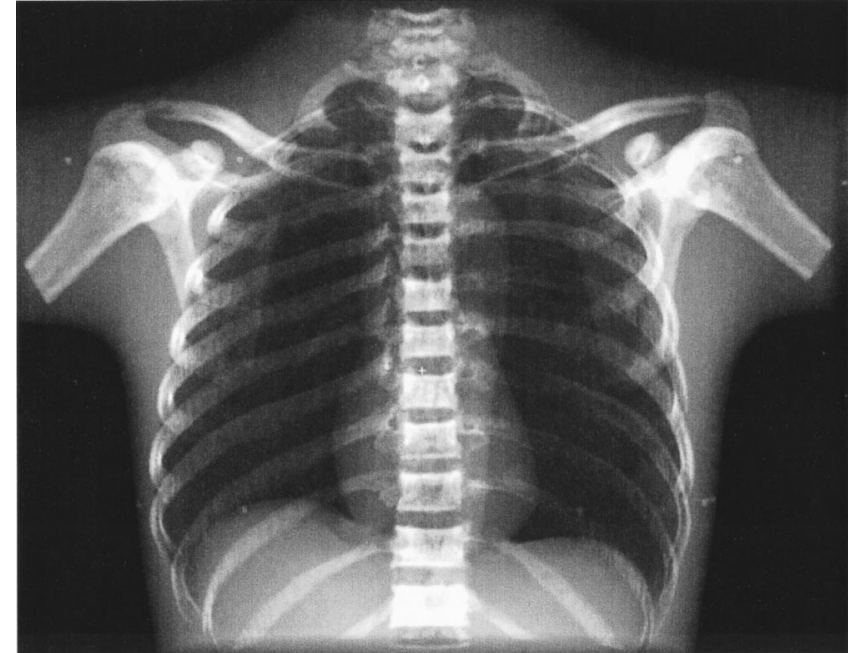

FIG. 1. A digitally reconstructed radiograph generated using $1 \mathrm{~mm}$ axial slices with $140 \mathrm{kV}$ and $340 \mathrm{~mA}$.

creasing slice thickness on DRR image quality. As expected, definition of features in the longitudinal direction decreases as slice thickness increases. Aside from this effect, all DRRs have sufficient contrast for definition of skeletal features, and clearly delineate the projections of the diaphragm and mediastinum.

Technique 4 shows image quality comparable to technique 1, and higher longitudinal resolution than techniques 2 and 3. There is no obvious degradation in the appearance of reference anatomical structures in this DRR due to the increased noise in the axial images resulting from the extremely low beam current used for scanning.

It is useful to mention the heat generated in producing these images. Technique 1 required 22.3 MHU for $\mathrm{x}$-ray production. Even with a 6.3 MHU capacity tube, cooling requirements limited scan acquisition time to $7 \mathrm{~s}$ per image, for a total scan acquisition time of $39 \mathrm{~min}$. By comparison, technique 2 required 3.4 MHU, technique 3 needed 2.0 MHU,

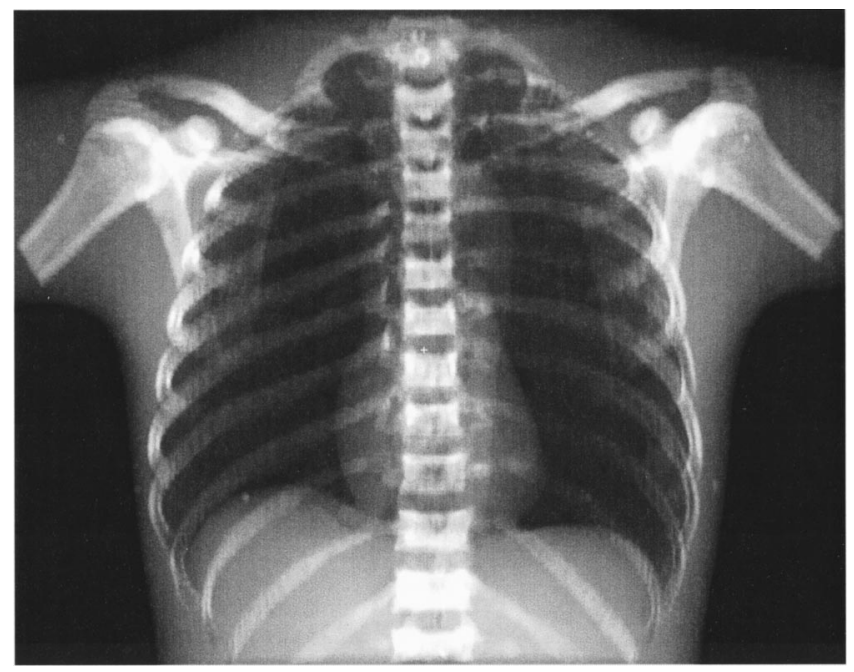

FIG. 2. A digitally reconstructed radiograph generated using $3 \mathrm{~mm}$ axial slices with $120 \mathrm{kV}$ and $180 \mathrm{~mA}$.

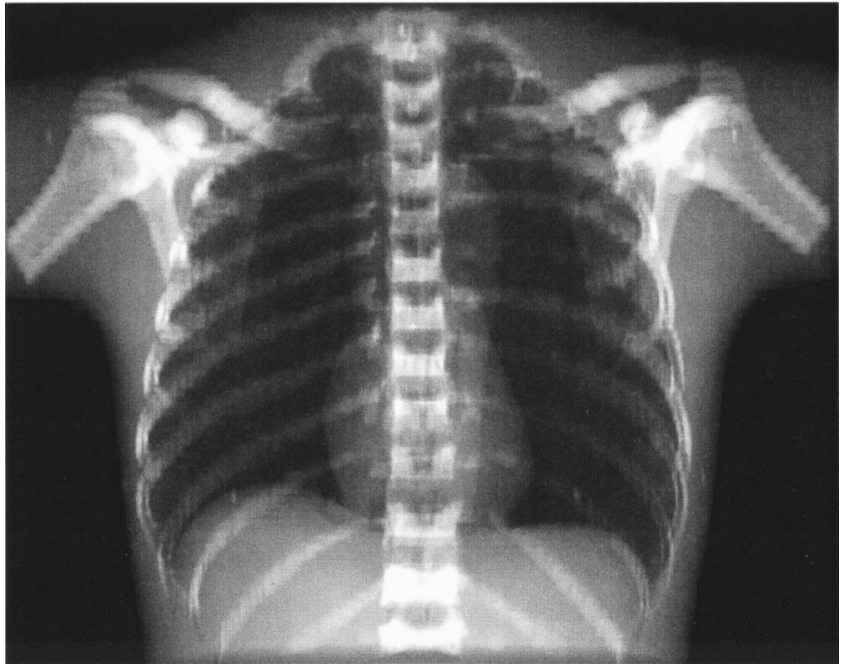

FIG. 3. A digitally reconstructed radiograph generated using $5 \mathrm{~mm}$ axial slices with $120 \mathrm{kV}$ and $180 \mathrm{~mA}$.

and technique 4 required $0.7 \mathrm{MHU}$. Techniques 2-4 did not exceed tube heating/cooling limitations, and could have been performed in helical mode instead of axial mode with no intrascan delays for cooling.

This simple demonstration shows that it is possible to separate the dual needs of acquiring high quality CT images to aid in treatment planning and acquiring high resolution $\mathrm{CT}$ models of the patient for generation of reference radiographs to aid in treatment setup and verification. By optimizing protocols for the acquisition and handling of CT data, a radiotherapy department could potentially reduce the technical requirements on CT hardware necessary for simulation.

Two modes of operation could potentially be considered to take advantage of these findings with conventional systems, with only minor modifications. If it is known that only a subregion of the scanned volume requires low noise to aid in volume definition, then the scanning protocol could involve acquisition with very low current through most of the

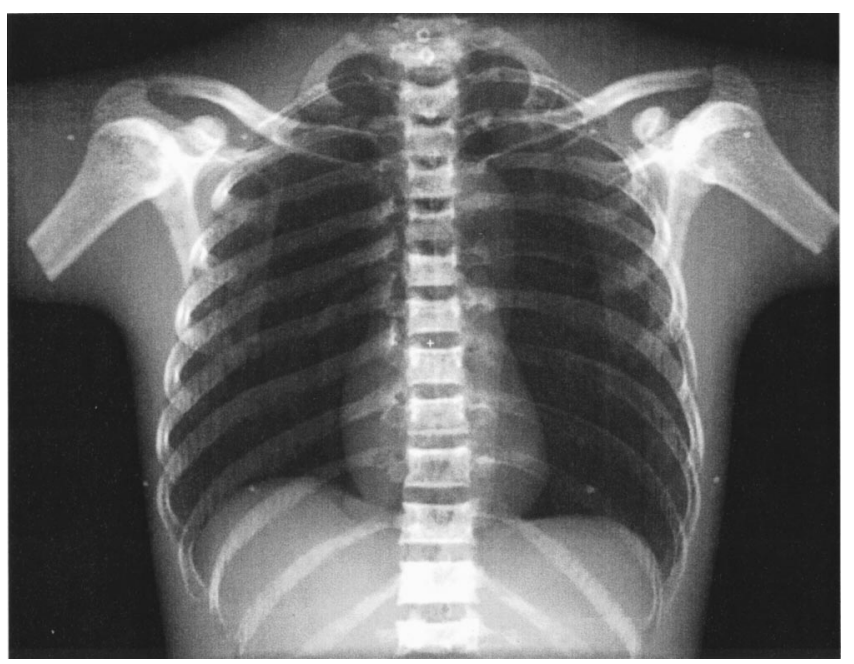

FIG. 4. A digitally reconstructed radiograph generated using $1 \mathrm{~mm}$ axial slices with $140 \mathrm{kV}$ and $10 \mathrm{~mA}$. 
volume, and increased current in the region of interest. A second mode of operation could involve the acquisition of two patient models, one at high resolution with extremely low current, and a second using a larger slice thickness at higher currents. As long as the treatment planning software is capable of handling these two volumes with the same coordinate system, it is possible to plan treatments with reduced data requirements, and to still present the plan and setup information with high resolution DRRs.

The authors wish to acknowledge Joanna Marry for help in CT data acquisition. This work was supported in part by
National Cancer institute Grant No. 2 P01 CA59827-06 and Grant No. 1 RO1 CA81161-01A1. J. M. B. is supported as a Kimmel scholar.

${ }^{a)}$ Electronic mail: jbalter@umich.edu

${ }^{1}$ J. Balter, R. K. Ten Haken, and K. L. Lam, "Treatment setup verification," in Teletherapy, Present and Future, edited by T. R. Mackie and J. R. Palta (Advanced Medical Publishing, Madison, WI, 1996), pp. 471493.

${ }^{2}$ M. Murphy, "The importance of computed tomography slice thickness in radiographic patient positioning for radiosurgery,' Med. Phys. 26, 171175 (1999). 